\title{
A novel complementary method for ultrasonographic screening of deep endometriosis: a case series of 5 patients diagnosed with transvaginal strain elastography
}

\author{
Gábor Szabó ${ }^{1, *}$, István Madár ${ }^{1}$, János Rigó Jr. ${ }^{1,2}$, Noémi Dobó ${ }^{1}$, Nándor Ács ${ }^{1}$, Attila Bokor ${ }^{1}$ \\ ${ }^{1}$ Department of Obstetrics and Gynecology, Faculty of Medicine, Semmelweis University, 1088 Budapest, Hungary \\ ${ }^{2}$ Department of Clinical Studies in Obstetrics and Gynecology, Faculty of Health Sciences, Semmelweis University, 1088 Budapest, Hungary \\ *Correspondence: szabo.gabor6@med.semmelweis-univ.hu (Gábor Szabó) \\ Academic Editor: Simone Ferrero \\ Submitted: 29 November 2020 Revised: 13 January 2021 Accepted: 19 January 2021 Published: 6 January 2022
}

\begin{abstract}
Background: Ultrasound elastography displays information on tissue stiffness. Deep endometriotic nodules are hard fibrotic tissues. Patients are recognized as having deep endometriosis only after several years from the onset of symptoms, therefore it is important to improve diagnostic capabilities. Cases: In this case series, our purpose was to present the applicability and feasibility of transvaginal strain elastography. Five patients with various complaints compatible with endometriosis underwent transvaginal ultrasound with strain elastography. Using the 'International Deep Endometriosis Analysis' group (IDEA) protocol along with transvaginal strain elastography, preoperative examination clearly demonstrated the size and extent of deep endometriosis. Conclusion: This ultrasonographic technique was effective regardless of whether the ligaments of the female reproductive tract, or the organs of the urinary and intestinal tract were infiltrated.
\end{abstract}

Keywords: Ultrasound elastography; Endometriosis; Strain elastography; IDEA protocol; Deep endometriosis; Laparoscopy

\section{Introduction}

The estimated overall prevalence of endometriosis is $7-12 \%$ in the general female population [1]. This condition is one of the leading causes of infertility with symptoms ranging from chronic catamenial pain, irregular bleeding or, dyspareunia all leading to decreased quality of life and potential infertility. The detection of endometriosis may be delayed by up to 7 years from presenting symptoms due to a lack of non-invasive diagnostic tools. The four classic phenotypes of the disease are adenomyosis affecting the uterine wall, endometrioma in the ovaries, superficial implants on the peritoneum and subperitoneal infiltration of $>5 \mathrm{~mm}$ into pelvic/abdominal organs defined as deep endometriosis (DE).

DE lesions in the pelvis can be palpated as hard fibrotic nodules [2] by bimanual pelvic examination. Glandular epithelial or active stromal cells are frequently absent histologically in deep endometriotic nodules, while metaplastic smooth muscle components and fibrosis are always present. Vigano et al. [3] have suggested a new definition of endometriosis being "a fibrotic condition in which endometrial stroma and epithelium can be identified".

MRI, colonoscopy and cystoscopy have been suggested for the detection of DE. For multiple reasons, transvaginal ultrasound has become the first line diagnostic tool for DE [4-6]. The precise description of an endometrioma is facilitated by the 'International Ovarian Tumor Analysis' (IOTA) criteria [7]. The sonographic fea- tures of adenomyosis are enumerated by the 'Morphological Uterus Sonographic Assessment' (MUSA) criteria [8,9], whereas diagnostic steps of DE are defined by the protocol of the IDEA group [10]. With experienced sonographers, the detection rate of $\mathrm{DE}$ can even reach 90 percent [11]. Although a significant proportion of these lesions are not detected [12].

Ultrasound elastography has been developed over the last three decades to display information on tissue stiffness $[13,14]$. Use of a transvaginal transducer produces a strain image by using the transducer to apply repetitive minimal pressure on the tissues being examined. Manufacturers offer several methods to generate feedback to the examiner on the degree of the applied compression by utilizing a strain graph display, bar, numerical value or scale. Visualizing the monitor provides real-time feedback about whether the appropriate amount of pressure is being applied. The subsequent tissue displacement is tracked between pairs of radiofrequency echo frames and the strain is calculated from the axial gradient of the displacements. Under an equal amount of stress, a stiff region undergoes less deformation than surrounding softer tissues. Strain ratio between two regions can then be calculated. Using a color map to code different magnitudes of strain, a two-dimensional strain image can be translucently superimposed on the conventional B-mode image, aiding the assessment of the spatial relationship between the ultrasound image and the elastographic data. If the required minimum pressure is reached during the examination, the color-coded two-dimensional map au- 
tomatically appears on the display. Blue color indicates hard tissue, yellow and green imply relative medium tissue stiffness and red color marks soft tissue, as confirmed by Stoelinga et al. [15]. We are not aware of any data using transvaginal strain elastography for systematic screening of deep endometriosis. Our objective was to visualize deep endometriotic lesions utilizing this technique.

In this case series, we present our results on the applicability and feasibility of transvaginal strain elastography to diagnose deep endometriotic lesions at different body sites. Informed consent was obtained from each patient and the Institutional Review Board approved our study.

\section{Case report}

\subsection{Case 1}

Transabdominal ultrasound performed in a 33-yearold woman (Fig. 1A). Demonstrated a $38 \times 24 \times 21 \mathrm{~mm}$ diameter solid and hypoechoic structure causing an irregular contour in the wall of the urinary bladder and protruding into the abdominal cavity. The transvaginal ultrasound scan demonstrated the hypoechoic lesion to be located at the base of the urinary bladder and adherent to the anterior uterine wall. Ultrasound strain elastography (Samsung WS80A with Elite, Seoul, South Korea, Elastoscan software and 5$9 \mathrm{MhZ}$ transvaginal probe) revealed the lesions stiffness to be harder than the urinary bladder wall and the surrounding tissues (Fig. 1B). The difference ratio was more than eightfold between the lesion and the normal bladder wall (Fig. 1C).

The suspicion of endometriosis arose due to the complaints and the age of the patient, as well as localization of the nodule. Based on the irregular contour, bladder cancer was also suspected. Secondary to a pregnancy in the previous year, deep endometriosis of the bladder may have undergone decidualization [16-18]. During laparoscopic surgery, a lesion measuring $3 \mathrm{~cm}$ diameter corresponding to deep infiltrating endometriosis was detected at the bladder base (Fig. 1D). In this case, strain elastography successfully distinguished deep endometriosis from bladder cancer.

\subsection{Case 2}

During a bimanual pelvic examination in a 26-yearold woman, a palpable painful stiff nodule $2 \mathrm{~cm}$ diameter was found in the posterior vaginal fornix. Using transvaginal ultrasonography, a hypoechoic region was detectable below the level of the lower border of the posterior lip of the cervix in the widened hyperechogenic rectovaginal septum (Fig. 2A). Sliding sign-which is an excellent dynamic marker against rectouterine pouch obliteration - was positive $[10,19,20]$. Using ultrasound strain elastography, the nodule presented as blue color. This is the typical appearance for hard tissue in the Elastoscan mode of the ultrasound device. The nodule measured $17 \times 12 \times 9 \mathrm{~mm}$. The wall of the rectum and the vagina were yellow and red suggesting soft appearance (Fig. 2B). The nodule stiffness was harder
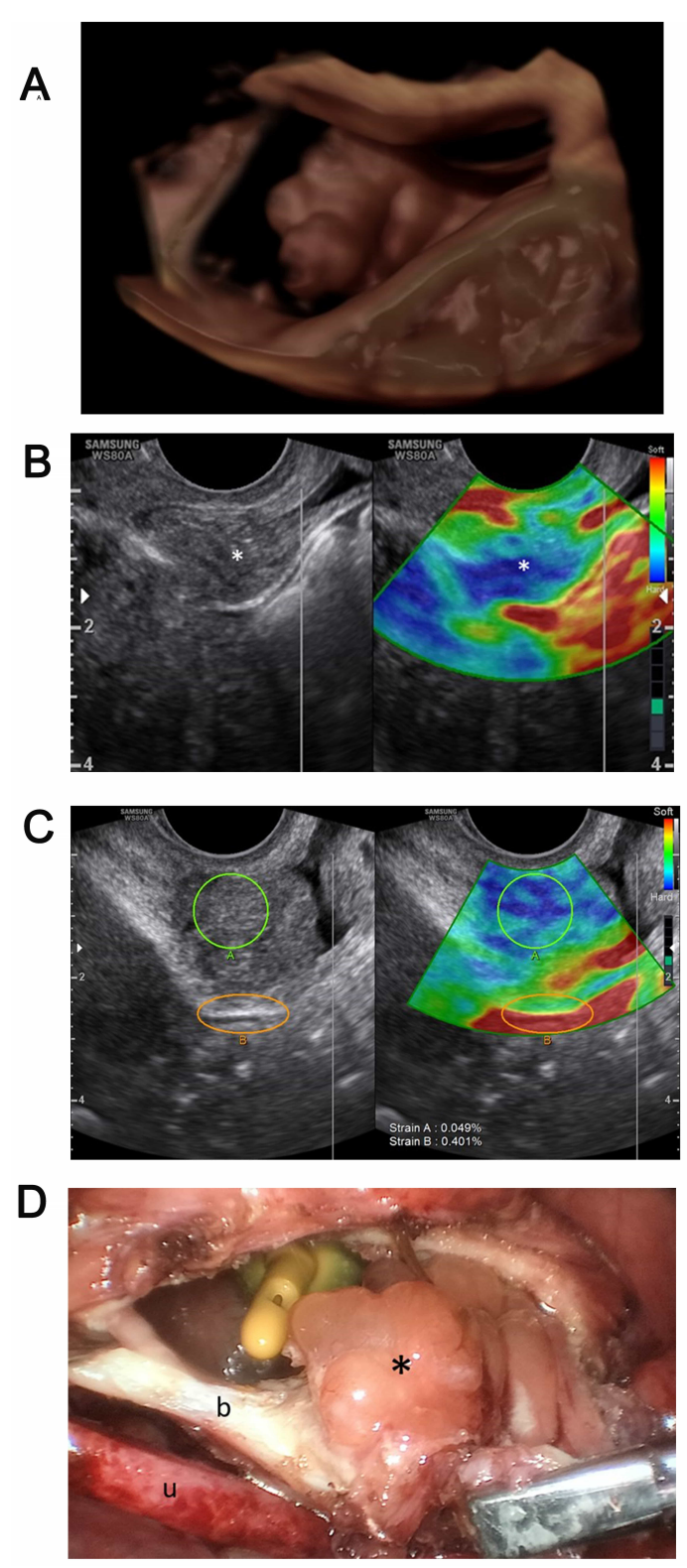

Fig. 1. Deep endometriosis of the urinary bladder in a 33 yearold woman. (A) A three-dimensional visualization of the bladder nodule with transabdominal scan and surface rendering. (B) Dualmode gray-scale sonographic (left) and strain elastography (right) images with transvaginal scan. An ascending-colors map is used: dark blue indicates harder tissue; green and yellow indicates moderately stiff tissue; and orange and red indicates soft tissue. The bar in the lower right corner of the screen indicates the pressure applied. A hypoechoic and hard structure (according to elastography) with irregular contour protrudes into the cavity of the bladder (asterisk). (C) Strain measurement between the lesion (green circle) and the normal bladder wall (orange circle). (D) Laparoscopic image of the bladder nodule (asterisk). Endometriosis infiltrates both the bladder (b) and uterine (u) walls.

than the hyperechoic regular connective tissue in the rectovaginal septum. The difference ratio was almost threefold 
between the hypoechoic lesion and the normal hyperechoic rectovaginal septum (Fig. 2C). Rectovaginal septum endometriosis which is rare dominantly starts from the upper third of the vagina [21]. In this case, neither the retroperitoneal rectum nor vaginal wall were infiltrated. The excision of a $2 \mathrm{~cm}$ nodule was performed (Fig. 2D). Histological examination confirmed deep endometriosis of the rectovaginal septum. During conventional B mode ultrasound examinations, fluid-containing lesions of the vaginal wall such as Gartner's duct cysts, mucous inclusions and epithelial inclusion cysts are hypoechogenic and show no Doppler flow. Strain elastography can adequately identify fibrotic, hard nodules of deep endometriosis in this location.

\subsection{Case 3}

During a bimanual examination in a 23-year-old woman, the left ovary was determined to be enlarged. Transvaginal ultrasound demonstrated the typical appearance of an ovarian endometrioma with a unilocular cyst of $4 \mathrm{~cm}$ diameter with ground-glass echogenicity and no papillary projections. Color-flow Doppler sonography did not show any flow in the cyst. Strain elastography made parts of the cystic lesion in the ovary clearly distinguishable with the supernatant being softer and the sediment denser than the normal ovarian tissue (Fig. 3A).

According to the second step of the IDEA protocol, we evaluated soft markers as site specific tenderness and ovarian mobility [22,23]. The patient did not report any pain. The left ovary was mobile medially against the uterus and the contralateral ovary but fixed inferomedially against the ipsilateral pelvic sidewall. Between the left uterosacral ligament and the left ovary, a hypoechogenic "triangle" was wisible with B mode. An elastographic examination was performed to distinguish between adhesions and deep endometriosis. At the junction of the uterosacral ligament and the ovarian cyst wall, a hard nodule with a diameter of $2 \mathrm{~cm}$ was identified with elastography (Fig. 3A). The sliding sign between the rectum and the uterine fundus was positive.

A laparoscopic cystectomy was performed. A small endometrioma (chocolate cyst) of the left ovary and the presence of adhesions were confirmed during the procedure (Fig. 3B). The endometrioma was fixated inferomedially to the ipsilateral uterosacral ligament. After dissecting the adhesions, a $2 \mathrm{~cm}$ deep endometriotic nodule of the left uterosacral ligament was removed (Fig. 3C). Strain elastography confirmed the presence of superficial and ovarian type of endometriosis along with the deeply infiltrating form.

\subsection{Case 4}

A 32-year-old nulligravid woman presented with left dorsal pain and hematuria. Renal ultrasonography showed dilation in the pelvis and calyces of the left kidney and left hydroureter. The right kidney and ureter appeared to be normal. Transvaginal ultrasonography verified that the to-
A

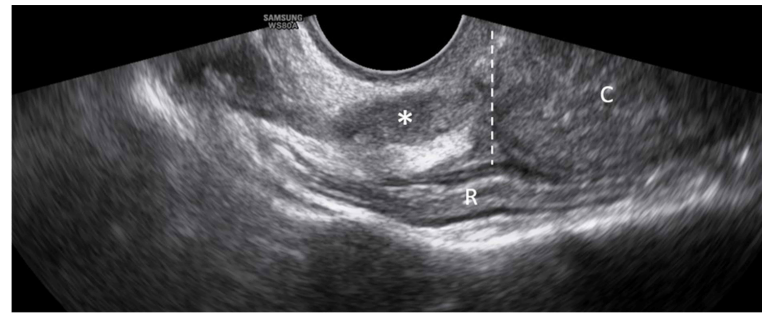

B

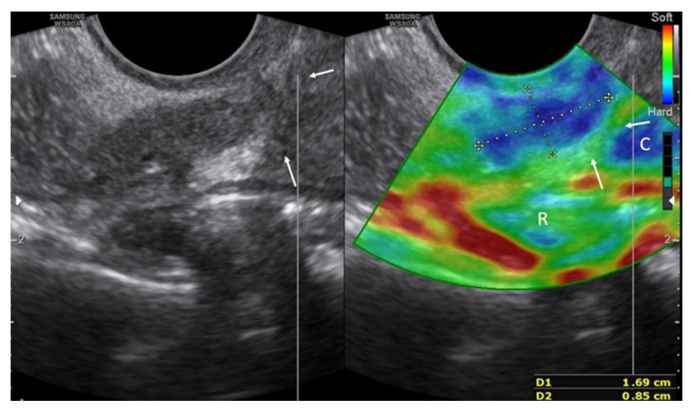

C

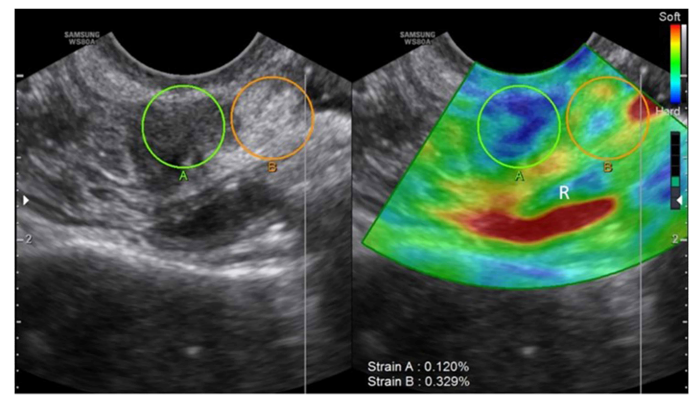

D

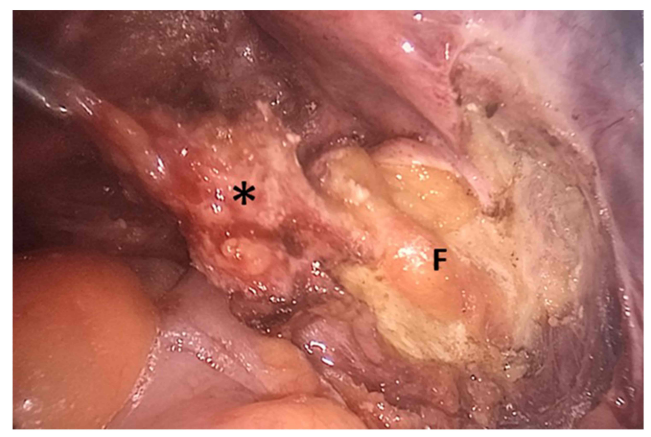

Fig. 2. Deep endometriosis of the rectovaginal septum in a 26 year-old woman. (A) Sagittal transvaginal sonogram showing a hypo-echoic mass (asterisk) below the level of the lower border of the posterior lip of the cervix (dashed line). ' $\mathrm{C}$ ' indicates cervix, ' $R$ ' indicates rectum. (B) Dual-mode gray-scale sonographic (left) and strain elastography (right) images with sagittal transvaginal scan. The sonoelastographic appearance of the endometriosis nodule shows blue color - hard stiffness (calipers). The wall of the rectum $(\mathrm{R})$ and the posterior vaginal fornix (arrows) are soft tissues (red, yellow and green). ' $\mathrm{C}$ ' indicates cervix. (C) Transverse transvaginal sonogram with strain measurement between the lesion (green circle) and the adjacent regular hyperechoic rectovaginal septum (orange circle). 'R' indicates rectum. (D) Laparoscopic image with excision of the deep endometriosis lesion (asterisk). Note the surrounding soft, fatty tissue in the rectovaginal septum. 
A

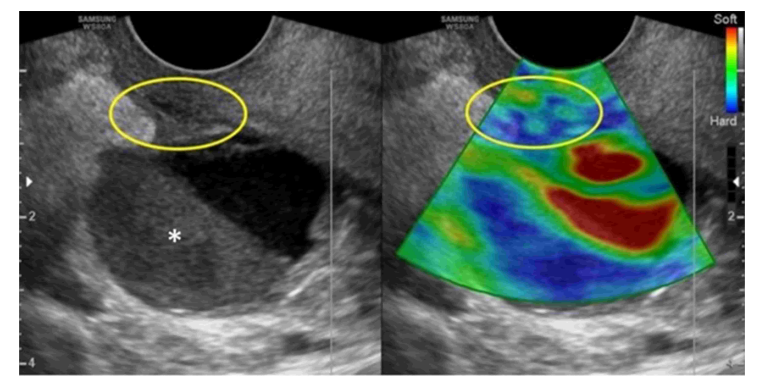

B

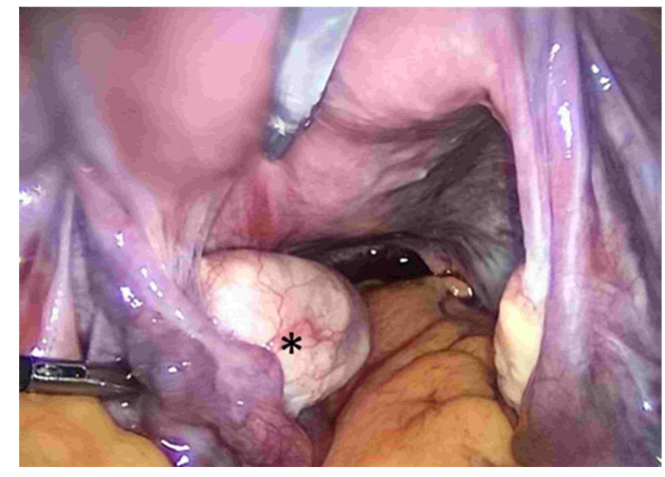

C

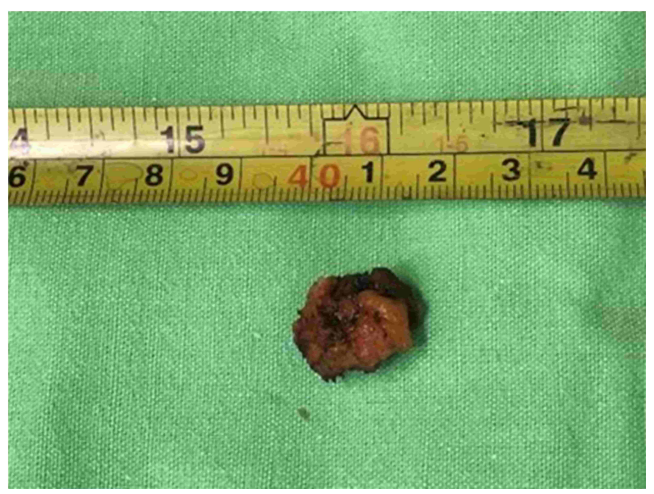

Fig. 3. Endometrioma in the left ovary and deep endometriosis of the left uterosacral ligament in a 23 year-old woman. (A) Dual-mode gray-scale sonographic (left) and strain elastography (right) images with transvaginal scan. A unilocular ovarian cyst is visualized with ground-glass echogenicity (asterisk). Using elastography, the sediment appears stiffer than the supernatant. Inferomedially a hypoechogenic triangle pulls the ovary toward the uterosacral ligament. Sonoelastographic appearance of this area shows blue color: hard stiffness (yellow circle). (B) Laparoscopic image. The left ovary is enlarged due the endometrioma (asterisk). There are adhesions present between the left ovary, the left fallopian tube and the left uterosacral ligament. (C) Excised deep endometriosis nodule from the left uterosacral ligament.

tal pelvic portion of the ureter showed a dilation of $9 \mathrm{~mm}$ in diameter [24-26]. The source of the obstruction was a hypoechoic nodule with a diameter of $3 \mathrm{~cm}$ in the left parametrium. It involved both the distal part of the left ureter and the trigone of the bladder (Fig. 4A). Ultrasound elastography demonstrated the nodule's stiffness to be more then twofold compared to the adjacent pelvic side wall stiffness (Fig. 4B).
Transvaginal ultrasonography identified another hypoechogenic nodule with a diameter of $9 \mathrm{~mm}$ infiltrating the left uterosacral ligament and torus uterinus (transverse ridge on the back part of the cervix of the uterus, formed by the junction of the uterosacral ligaments) [27]. This nodule was adherent to the anterior wall of the rectum with the layer of the muscularis externa appeared to be intact. Ultrasound elastography demonstrated the nodule's stiffness to be almost threefold compared to the adjacent pelvic side wall stiffness (Fig. 4C).

Laparoscopy revealed the DE nodule infiltrating the juxtavesical left ureter extending to the left ureteral orifice and trigone of the urinary bladder. Ultrasound strain elastography assisted in to differentiating the intrinsic type ureteral deep endometriosis from other tumors causing ureteral stenosis.

\subsection{Case 5}

A 36 year-old nulligravid woman presented with dysmenorrhoea, catamenial bloating, diarrhea and haematochezia. During pelvic examination the uterus appeared fixed and painful. A transvaginal scan on the sagittal view showed that the cervix was anteverted (directed anteriorly towards the urinary bladder) with the endometrium of the uterine fundus being deviated versus the pelvis posterior compartment. This sonographic sign called 'Question mark sign' is a marker for adenomyosis and strongly suggests the presence of deep endometriosis in the posterior compartment $[10,28]$. The posterior wall of the uterus was threefold thicker than the anterior wall. In addition to the asymmetrical thickening, a hyperechoic island were also depicted in the myometrium (Fig. 5A). A hypoechoic anterior rectal lesion fixed to the posterior uterine fundus was visible. This mushroom cap shaped nodule of $38 \times 32 \times 30 \mathrm{~mm}$ obstructed the lumen of the rectum. The lesion changed the normal appearance of the muscularis propria layer of the rectum [29]. The thin hypoechoic line was replaced by an irregular thick lesion with retraction and adhesions, resulting in the so-called 'Indian headdress' sign [10]. The nodule had a small tail at the distal end (comet tail) [30] (Fig. 5B). The distance between the lesion's distal end and the anal verge was $12 \mathrm{~cm} \mathrm{[31].} \mathrm{During} \mathrm{ultrasound} \mathrm{strain}$ elastography, the lesion was clearly demarkated both in sagittal (Fig. 5C) and transverse plane. The posterior vaginal fornix and the left uterosacral ligament were also affected by the nodule (Fig. 5D). Ultrasound strain elastography provided additional information on the extent of deep endometriosis. The stiffness of the thickened anterior rectal wall was harder than the regular posterior wall with the difference ratio being more than threefold (Fig. 5E). The surgically removed specimen was a $4 \mathrm{~cm}$ diameter nodule protruding into the bowel cavity (Fig. 5F). 
A

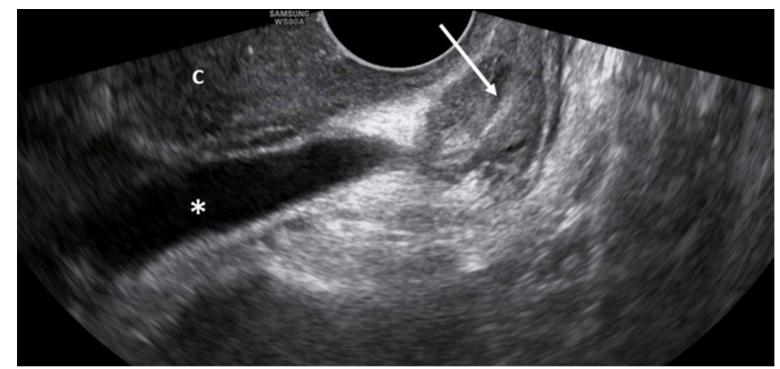

B

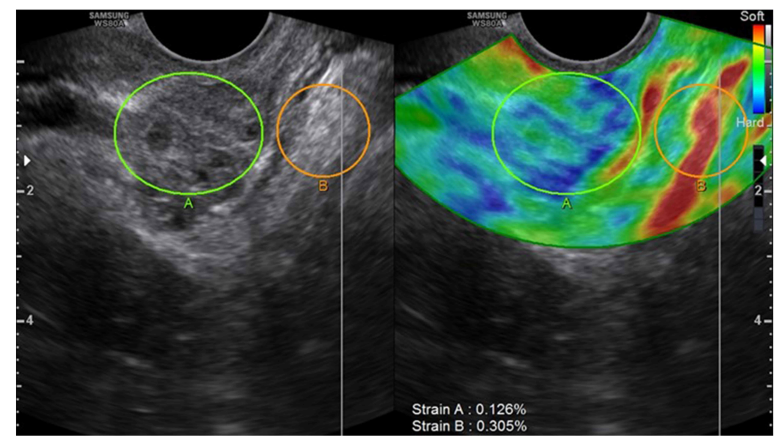

C

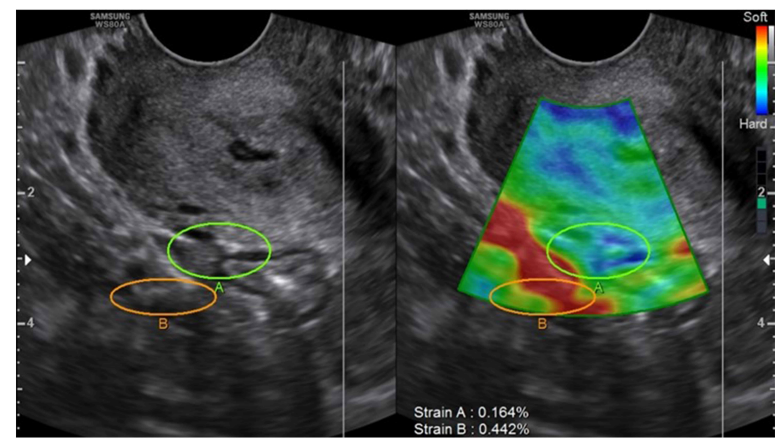

Fig. 4. Left hydrooureter caused by intrinsic left ureteral and bladder deep endometriosis in a 32 year-old woman. (A) Transvaginal scan of the left parametrium showing a dilated ureter as a tubular structure with anechoic content (asterisk). At the distal part of the left ureter, a bulky obstructing lesion involving the trigone of the bladder also was present (arrow). ' $\mathrm{C}$ ' indicates cervix. (B) Transvaginal scan of the left parametrium. Strain measurement between the lesion (green circle) and the adjacent pelvic sidewall (orange circle). (C) Transverse image at the level of the upper cervix. A hypoechogenic nodule in the midline, at the torus uterinus is attached to anterolateral wall of the rectum. The layer of the muscularis propria is not thickened. Sonoelastographic appearance of the lesion showing stiff tissue (green circle) in the soft pararectal environment (orange circle).

\section{Discussion}

Endometriosis is a heterogeneous with various symptoms that do not necessarily correlate with the location of the endometriosis making this an enigmatic disorder. Deep endometriosis is commonly confirmed by laparoscopy [32]. In many cases, if the surgery is not performed by a specialist or if the proper technical conditions are not met, a second intervention is required [33]. The patient is often overbur- dened and the financial implications are significant [34]. To avoid these repeated surgeries, a number of imaging techniques are used in preoperative evaluations. The availability of magnetic resonance imaging is limited and not all radiologists have experience in the field of endometriosis $[35,36]$. Other modalities such as colonoscopy and cystoscopy are invasive and have low diagnostic accuracy [37].

Ultrasound plays a critical role in the diagnosis and surgical management decision-making for endometriosis patients [38,39]. Modified ultrasound techniques like gel sonovaginography and rectal water contrast transvaginal ultrasonography are also complex methods although they improve diagnostic performance [40,41].

Previously, patients with endometriosis were studied with elastography, mainly for adenomyosis and ovarian endometriomas. With shear wave elastography endometriomas have higher levels of stiffness compared to hemorrhagic ovarian cysts [42]. Case 3 confirmed this observation. In the uterus, adenomyotic areas presented more softness (red and green color) compared with the surrounding uterine tissue (blue color) determined by transvaginal strain elastography. The borders of the adenomyotic areas corresponded to the borders of the green area [43]. These results confirm the histologic fact that infiltration of the glandular tissue into the myometrium, the repetitive hemorrhages and inflammatory processes damage the integrity of the uterine wall. The addition of strain elastography to gray-scale ultrasound appears to be useful in the differentiation between fibroids, adenomyosis and normal uteri [44].

Ding et al. [45] utilized shear wave elastography to examine pelvic endometriosis nodules. They reported that lesional stiffness obtained with their method, correlated closely and positively with the extent of lesional fibrosis in histologic specimens. Their article did not include a pictorial presentation of the method or its implementation.

Transvaginal strain elastography uses the characteristic pathophysiologic process of deep endometriosis, such as scarring and the formation of dense fibrous connective tissue [46]. The examination does not require any preparation and does not involve any differences for the patient than the transvaginal ultrasound examination [14]. The applied repetitive minimal pressure on the pelvic organs during elastography is in accordance with the "Tendernessguided" transvaginal ultrasonography soft marker, also incorporated in the IDEA protocol [10].

The acquired color map makes the lesions clearly visible both to the examiner and to the patient. This helps the surgeon determine the size and extent of endometriosis and to organize the appropriate minimally invasive gynecologic surgical (MIGS) team [47]. The patient becomes more aware of the pathology behind their complaints so that preoperative counseling can become more thorough. In our opinion, just as the use of color-Doppler has significantly aided development of echocardiography, elastography may assist in the diagnosis of deep endometriosis. 
A
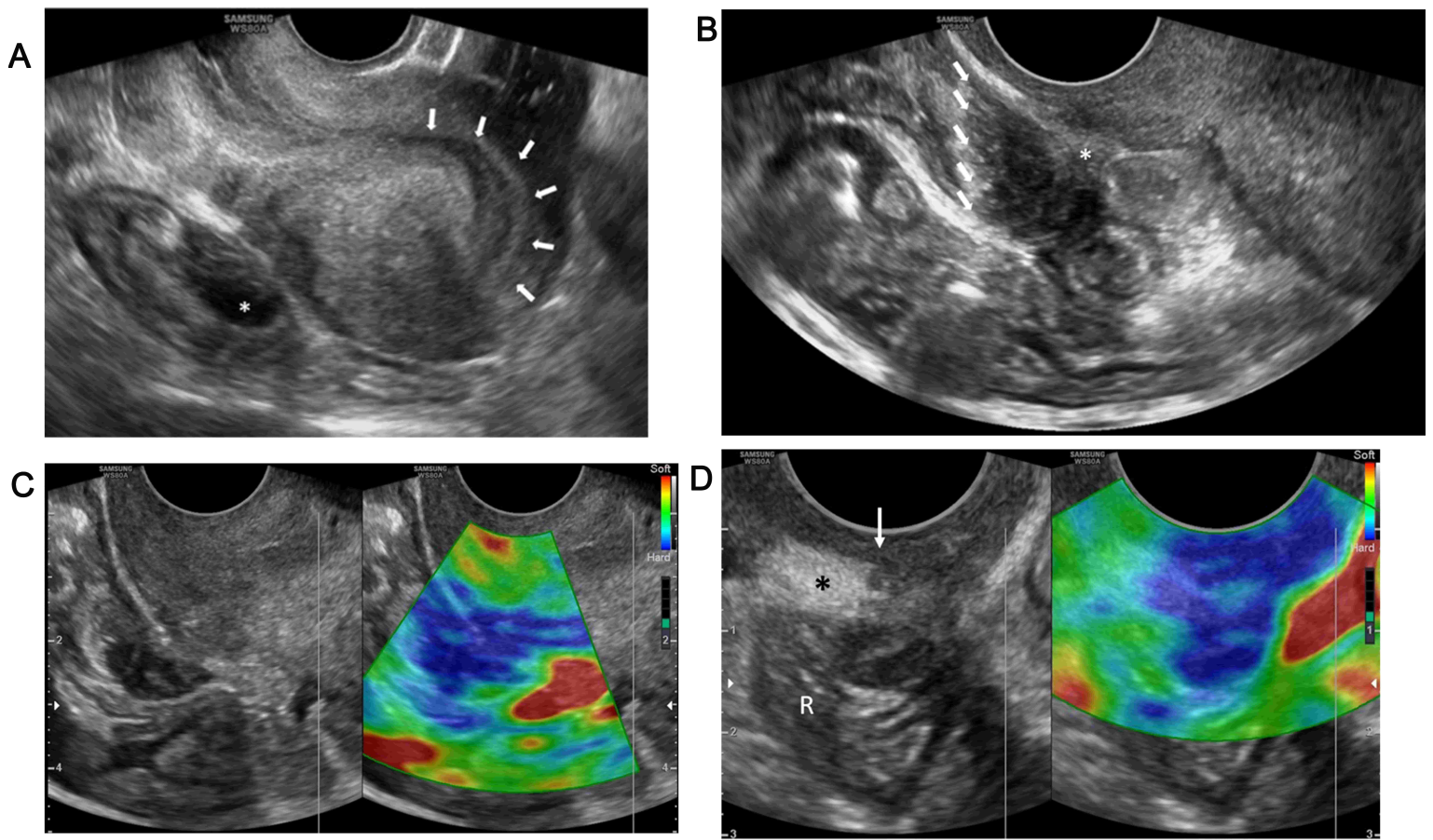

E
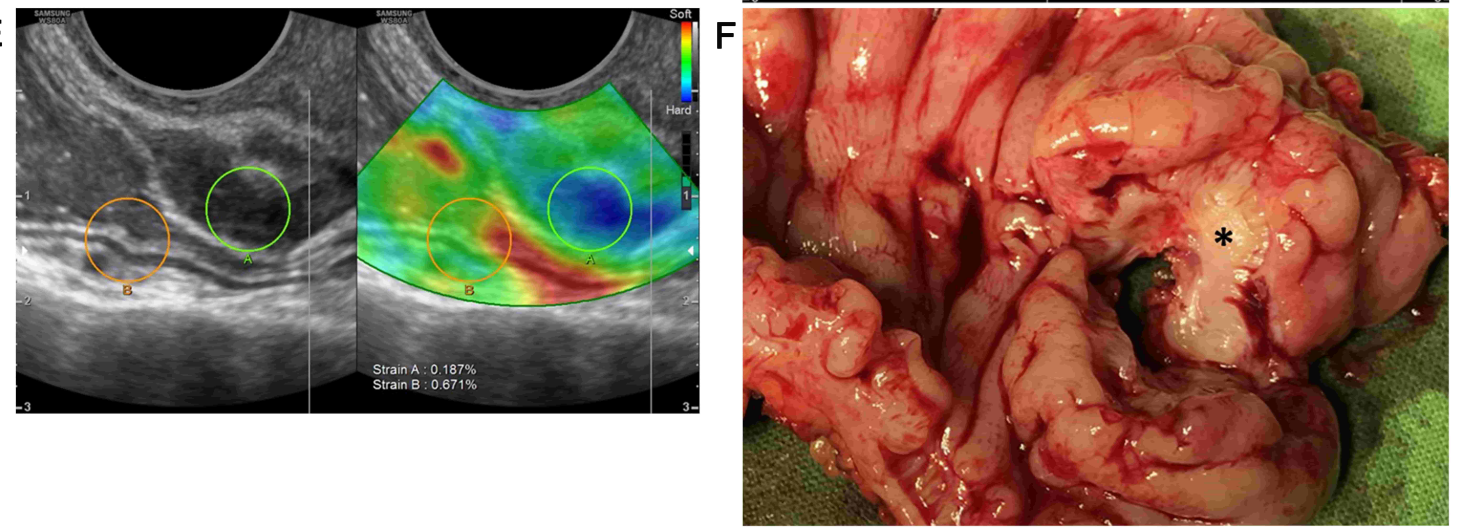

Fig. 5. Rectal deep endometriosis and adenomyosis in a 36 year-old woman. (A) Transvaginal scan on the sagittal view. Note the deviated axis of the uterine cavity in the fundus (arrows). This sonographic sign is called 'Question mark sign'. The posterior wall of the uterus is threefold thicker than the anterior wall suggesting adenomyosis. A hypoechoic anterior rectal lesion fixed to the posterior uterine fundus is visible (asterisk). (B) The irregular thick lesion (asterisk) interrupted the hyperechoic peritoneal layer of the pouch of Douglas causing retraction and adhesions (arrows), the so-called 'Indian headdress' sign in the anterior rectal wall. (C) Dual-mode grayscale sonographic (left) and strain elastography (right) sagittal images with transvaginal scan. The lesion is a clearly demarkable stiff tissue. (D) Dual-mode gray-scale sonographic (left) and strain elastography (right) transverse images with transvaginal scan. The lesion infiltrates the rectum, the hyperechoic uterosacral ligament (asterisk) and the hypoechoic vaginal wall (arrow). ' $R$ ' indicates rectum. (E) Transvaginal scan of the rectum. Transducer is in the posterior fornix. Strain measurement between the lesion in the anterior rectal wall (green circle) and the regular posterior rectal wall (orange circle). (F) Surgically removed specimen. The deep endometriosis nodule (asterisk) protrudes into the bowel cavity.

In our case series, we did not study the effect of medical treatment on the stiffness of deep endometriotic nodules because all surgeries were performed within 6 months of recognition. None of the patients received hormonal treatment during this period.

This method can be a valuable tool in the differential diagnosis of pelvic deep endometriosis. Improving transvaginal ultrasonography with strain elastography may assist in ruling out concomittant or alternative conditions $[48,49]$. For example fluid-containing vaginal wall cysts, rectal and bladder cancers, inflammatory bowel disease, or perianal abscesses may become more easily distinguishable from deep endometriotic nodules. Based on previous ultrasound elastographic examinations, benign adenomas of the bowel show no significant difference compared to normal intestinal wall thickness. Rectal cancer at different stages 
have increased stiffness values [50]. The application of strain elastography to distinguish between rectal cancer and deep endometriosis lesions infiltrating the rectum should be the subject of further studies. Endometriosis is associated with similar symptoms to Crohn's disease, a common inflammatory bowel disease among young women of reproductive age. In Crohn's disease with intestinal strictures and fibrosis, diffuse elevated stiffness was detected by elastographic examination [51]. Further prospective studies may clarify the potential role of elastography in the differential diagnosis of these two diseases.

\section{Conclusions}

In summary, we have described and demonstrated the effectiveness of transvaginal strain elastography in the preoperative assessment of patients with DE. Using steps of the IDEA protocol supplemented with strain elastography, endometriosis nodules with different locations were clearly detectable in the patients studied. The method is suitable for accurate assessment of the extent of pelvic deep endometriosis. Based on strain ratios, the hardness of the nodules was a multiple of the healthy tissues. Laparoscopic surgery and histologic examination confirmed the findings of the transvaginal ultrasonography in all cases.

Further studies are needed to determine the location and deep endometriosis nodule size for which the ultrasonographic diagnostic performance with this method can be improved.

\section{Abbreviations}

DE, deep endometriosis; IDEA, International Deep Endometriosis Analysis; IOTA, International Ovarian Tumor Analysis; MUSA, Morphological Uterus Sonographic Assessment.

\section{Author contributions}

GS, JR Jr and NA designed the research study. GS performed the sonography. IM provided help in the administration and data analysis. $\mathrm{ND}$ and $\mathrm{AB}$ performed the laparoscopic operations. GS wrote the manuscript. All authors contributed to editorial changes in the manuscript. All authors read and approved the final manuscript.

\section{Ethics approval and consent to participate}

Informed consent was obtained from each patient and the Institutional Review Board approved our study (58723/2016 EKU).

\section{Acknowledgment}

Thanks to all the peer reviewers for their opinions and suggestions.

\section{Funding}

This research received no external funding.

\section{Conflict of interest}

The authors declare no conflict of interest. JR Jr is the Editorial board member of this journal, given his role as Editorial board member, JR Jr had no involvement in the peer-review of this article and has no access to information regarding its peer-review.

\section{References}

[1] Vercellini P, Viganò P, Somigliana E, Fedele L. Endometriosis: pathogenesis and treatment. Nature Reviews Endocrinology. 2014; 10: 261-275.

[2] Hudelist G, Oberwinkler KH, Singer CF, Tuttlies F, Rauter G, Ritter $\mathrm{O}$, et al. Combination of transvaginal sonography and clinical examination for preoperative diagnosis of pelvic endometriosis. Human Reproduction. 2009; 24: 1018-1024.

[3] Vigano P, Candiani M, Monno A, Giacomini E, Vercellini P, Somigliana E. Time to redefine endometriosis including its profibrotic nature. Human Reproduction. 2018; 33: 347-352.

[4] Guerriero S, Alcázar JL, Pascual MA, Ajossa S, Perniciano $\mathrm{M}$, Piras A, et al. Deep infiltrating endometriosis: comparison between 2-dimensional ultrasonography (US), 3-dimensional US, and magnetic resonance imaging. Journal of Ultrasound in Medicine. 2018; 37: 1511-1521.

[5] Guerriero S, Saba L, Pascual MA, Ajossa S, Rodriguez I, Mais $\mathrm{V}$, et al. Transvaginal ultrasound vs magnetic resonance imaging for diagnosing deep infiltrating endometriosis: systematic review and meta-analysis. Ultrasound in Obstetrics and Gynecology. 2018; 51: 586-595.

[6] Vitagliano A, Andrisani A, Dessole F, Virdis G, Cappadona R, Marin L, et al. Rectovaginal endometriosis: head to head comparison between sonovaginography and magnetic resonance imaging. Clinical and Experimental Obstetrics \& Gynecology. 2019, 46: 888-891.

[7] Van Holsbeke C, Van Calster B, Guerriero S, Savelli L, Paladini $\mathrm{D}$, Lissoni AA, et al. Endometriomas: their ultrasound characteristics. Ultrasound in Obstetrics and Gynecology. 2010; 35: 730-740.

[8] Sakhel K, Abuhamad A. Sonography of adenomyosis. Journal of Ultrasound in Medicine. 2012; 31: 805-808.

[9] Van den Bosch T, Dueholm M, Leone FPG, Valentin L, Rasmussen CK, Votino A, et al. Terms, definitions and measurements to describe sonographic features of myometrium and uterine masses: a consensus opinion from the Morphological Uterus Sonographic Assessment (MUSA) group. Ultrasound in Obstetrics \& Gynecology. 2015; 46: 284-298.

[10] Guerriero S, Condous G, van den Bosch T, Valentin L, Leone FPG, Van Schoubroeck D, et al. Systematic approach to sonographic evaluation of the pelvis in women with suspected endometriosis, including terms, definitions and measurements: a consensus opinion from the International Deep Endometriosis Analysis (IDEA) group. Ultrasound in Obstetrics \& Gynecology. 2016; 48: 318-332.

[11] Hudelist G, English J, Thomas AE, Tinelli A, Singer CF, Keckstein J. Diagnostic accuracy of transvaginal ultrasound for noninvasive diagnosis of bowel endometriosis: systematic review and meta-analysis. Ultrasound in Obstetrics \& Gynecology. 2011; 37: 257-263.

[12] Fraser MA, Agarwal S, Chen I, Singh SS. Routine vs. expertguided transvaginal ultrasound in the diagnosis of endometriosis: a retrospective review. Abdominal Imaging. 2015; 40: $587-$ 594.

[13] Varghese T. Quasi-static ultrasound elastography. Ultrasound Clinics. 2009; 4: 323-338.

[14] Wells PNT, Liang H. Medical ultrasound: imaging of soft tis- 
sue strain and elasticity. Journal of the Royal Society, Interface. 2011; 8: 1521-1549.

[15] Stoelinga B, Hehenkamp WJK, Brölmann HAM, Huirne JAF. Real-time elastography for assessment of uterine disorders. Ultrasound in Obstetrics \& Gynecology. 2014; 43: 218-226.

[16] Frühauf F, Fanta M, Burgetová A, Fischerová D. Endometriosis in pregnancy - diagnostics and management. Ceska Gynekologie. 2019; 84: 61-67. (In Czech)

[17] Clement PB. The pathology of endometriosis: a survey of the many faces of a common disease emphasizing diagnostic pitfalls and unusual and newly appreciated aspects. Advances in Anatomic Pathology. 2007; 14: 241-260.

[18] Banner H, Murji A. Bladder endometriosis in pregnancy. American Journal of Obstetrics and Gynecology. 2020; 223: 450.

[19] Hudelist G, Fritzer N, Staettner S, Tammaa A, Tinelli A, Sparic $\mathrm{R}$, et al. Uterine sliding sign: a simple sonographic predictor for presence of deep infiltrating endometriosis of the rectum. Ultrasound in Obstetrics \& Gynecology. 2013; 41: 692-695.

[20] Reid S, Condous G. Transvaginal sonographic sliding sign: accurate prediction of pouch of Douglas obliteration. Ultrasound in Obstetrics \& Gynecology. 2013; 41: 605-607.

[21] Reid S, Condous G. The issues surrounding the pre-operative TVS diagnosis of rectovaginal septum endometriosis. Australasian Journal of Ultrasound in Medicine. 2014; 17: 2-3.

[22] Guerriero S, Ajossa S, Gerada M, D'Aquila M, Piras B, Melis GB. "Tenderness-guided" transvaginal ultrasonography: a new method for the detection of deep endometriosis in patients with chronic pelvic pain. Fertility and Sterility. 2007; 88: 1293-1297.

[23] Reid S, Leonardi M, Lu C, Condous G. The association between ultrasound-based 'soft markers' and endometriosis type/location: a prospective observational study. European Journal of Obstetrics, Gynecology, and Reproductive Biology. 2019; 234: 171-178.

[24] Pateman K, Mavrelos D, Hoo W, Holland T, Naftalin J, Jurkovic D. Visualization of ureters on standard gynecological transvaginal scan: a feasibility study. Ultrasound in Obstetrics \& Gynecology. 2013; 41: 696-701.

[25] Carfagna P, De Cicco Nardone C, De Cicco Nardone A, Testa AC, Scambia G, Marana R, et al. Role of transvaginal ultrasound in evaluation of ureteral involvement in deep infiltrating endometriosis. Ultrasound in Obstetrics \& Gynecology. 2018; 51: $550-555$.

[26] Aas-Eng MK, Salama M, Sevelda U, Ruesch C, Nemeth Z, Hudelist G. Learning curve for detection of pelvic parts of ureters by transvaginal sonography: feasibility study. Ultrasound in Obstetrics \& Gynecology. 2020; 55: 264-268.

[27] Leonardi M, Martins WP, Espada M, Arianayagam M, Condous G. Proposed technique to visualize and classify uterosacral ligament deep endometriosis with and without infiltration into parametrium or torus uterinus. Ultrasound in Obstetrics \& Gynecology. 2020; 55: 137-139.

[28] Di Donato N, Bertoldo V, Montanari G, Zannoni L, Caprara G, Seracchioli R. Question mark form of uterus: a simple sonographic sign associated with the presence of adenomyosis. Ultrasound in Obstetrics \& Gynecology. 2015; 46: 126-127.

[29] Hudelist G, Tuttlies F, Rauter G, Pucher S, Keckstein J. Can transvaginal sonography predict infiltration depth in patients with deep infiltrating endometriosis of the rectum? Human Reproduction. 2009; 24: 1012-1017.

[30] Benacerraf BR, Groszmann Y, Hornstein MD, Bromley B. Deep infiltrating endometriosis of the bowel wall. Journal of Ultrasound in Medicine. 2015; 34: 537-542.

[31] Aas-Eng MK, Dauser B, Lieng M, Diep LM, Leonardi M, Condous G, et al. Transvaginal sonography accurately predicts lesion to anal verge distance in women with deep endometriosis of the rectosigmoid. Ultrasound in Obstetrics and Gynecology.
2020; 56: 766-772.

[32] Leonardi M, Singh SS, Murji A, Satkunaratnam A, Atri M, Reid $\mathrm{S}$, et al. Deep endometriosis: a diagnostic dilemma with significant surgical consequences. Journal of Obstetrics and Gynaecology Canada. 2018; 40: 1198-1203.

[33] Menakaya UA, Rombauts L, Johnson NP. Diagnostic laparoscopy in pre-surgical planning for higher stage endometriosis: is it still relevant? Australian and New Zealand Journal of Obstetrics and Gynaecology. 2016; 56: 518-522.

[34] Ratner RT, Harris A, Tsaltas J, Goyal N, Davies-Tuck M, Najjar $\mathrm{H}$, Bare $\mathrm{O}$. An eight-year retrospective analysis of laparoscopic surgery for endometriosis, outcomes and complications in a large multicenter unit. Clinical and Experimental Obstetrics \& Gynecology. 2019; 46: 699-703.

[35] Leone Roberti Maggiore U, Biscaldi E, Vellone VG, Venturini PL, Ferrero S. Magnetic resonance enema vs rectal watercontrast transvaginal sonography in diagnosis of rectosigmoid endometriosis. Ultrasound in Obstetrics \& Gynecology. 2017; 49: 524-532.

[36] Berger JP, Rhemrev J, Smeets M, Henneman O, English J, Jansen FW. Limited added value of magnetic resonance imaging after dynamic transvaginal ultrasound for preoperative staging of endometriosis in daily practice: a prospective cohort study. Journal of Ultrasound in Medicine. 2019; 38: 989-996.

[37] Lukovich P, Csibi N, Brubel R, Tari K, Csuka S, Harsányi L, et al. Prospective study to determine the diagnostic sensitivity of sigmoidoscopy in bowel endometriosis. Orvosi Hetilap. 2017; 158: 264-269. (In Hungarian)

[38] Tammaa A, Fritzer N, Lozano P, Krell A, Salzer H, Salama M, et al. Interobserver agreement and accuracy of non-invasive diagnosis of endometriosis by transvaginal sonography. Ultrasound in Obstetrics \& Gynecology. 2015; 46: 737-740.

[39] Deslandes A, Parange N, Childs JT, Osborne B, Bezak E. Current status of transvaginal ultrasound accuracy in the diagnosis of deep infiltrating endometriosis before surgery: a systematic review of the literature. Journal of Ultrasound in Medicine. 2020; 39: 1477-1490.

[40] Reid S, Lu C, Hardy N, Casikar I, Reid G, Cario G, et al. Office gel sonovaginography for the prediction of posterior deep infiltrating endometriosis: a multicenter prospective observational study. Ultrasound in Obstetrics \& Gynecology. 2014; 44: 710 718.

[41] Bazot M, Malzy P, Cortez A, Roseau G, Amouyal P, Daraï E. Accuracy of transvaginal sonography and rectal endoscopic sonography in the diagnosis of deep infiltrating endometriosis. Ultrasound in Obstetrics \& Gynecology. 2007; 30: 994-1001.

[42] Batur A, Yavuz A, Ozgokce M, Bora A, Bulut MD, Arslan H, et $a l$. The utility of ultrasound elastography in differentiation of endometriomas and hemorrhagic ovarian cysts. Journal of Medical Ultrasonics. 2016; 43: 395-400.

[43] Tessarolo M, Bonino L, Camanni M, Deltetto F. Elastosonography: a possible new tool for diagnosis of adenomyosis? European Radiology. 2011; 21: 1546-1552.

[44] Stoelinga B, Hehenkamp WJK, Nieuwenhuis LL, Conijn MMA, van Waesberghe JHTM, Brölmann HAM, et al. Accuracy and reproducibility of sonoelastography for the assessment of fibroids and adenomyosis, with magnetic resonance imaging as reference standard. Ultrasound in Medicine \& Biology. 2018; 44: 1654 1663.

[45] Ding D, Chen Y, Liu X, Jiang Z, Cai X, Guo S. Diagnosing deep endometriosis using transvaginal elastosonography. Reproductive Sciences. 2020; 27: 1411-1422.

[46] Xie M, Zhang X, Zhan J, Ren Y, Wang W. Potential role of strain elastography for detection of the extent of large-scar endometriosis. Journal of Ultrasound in Medicine. 2013; 32: 1635-1642. 
[47] Exacoustos C, Malzoni M, Di Giovanni A, Lazzeri L, Tosti C, Petraglia F, et al. Ultrasound mapping system for the surgical management of deep infiltrating endometriosis. Fertility and Sterility. 2014; 102: 143-150.e2.

[48] Scioscia M, Orlandi S, Trivella G, Portuese A, Bettocchi S, Pontrelli $\mathrm{G}$, et al. Sonographic differential diagnosis in deep infiltrating endometriosis: the bowel. BioMed Research International. 2019; 2019: 5958402.

[49] Scioscia M, Zanetti I, Raspanti X, Spoto E, Portuese A, Noventa $\mathrm{M}$, et al. Ultrasound differential diagnosis in deep infiltrating endometriosis of the urinary tract. Journal of Ultrasound in Medicine. 2020; 39: 2261-2275.

[50] Havre RF, Leh S, Gilja OH, Ødegaard S, Waage JE, Baatrup G, et al. Strain assessment in surgically resected inflammatory and neoplastic bowel lesions. Ultraschall in Der Medizin. 2014; 35: $149-158$.

[51] Serra C, Rizzello F, Pratico' C, Felicani C, Fiorini E, Brugnera $\mathrm{R}$, et al. Real-time elastography for the detection of fibrotic and inflammatory tissue in patients with stricturing Crohn's disease. Journal of Ultrasound. 2017; 20: 273-284. 